\title{
An Approach to solve Hexagonal Fuzzy Assignment Problem using Modified Best Candidate Method and Comparative Study with Existing Methods
}

\author{
${ }^{1}$ M.Maragatham \\ ${ }^{1}$ Associate Professor of Mathematics, $P G$ and Research Department of Mathematics, Periyar \\ E.V.R College (Autonomous)*,Tiruchirappalli, \\ *Affiliated to Bharathidasan University
}

\author{
Suzane Raj.L \\ ${ }^{2}$ Research Scholar, \\ $P G$ and Research Department of Mathematics, \\ Periyar E.V.R College (Autonomous)*Tiruchirappalli, \\ *Affiliated to Bharathidasan University
}

\begin{abstract}
The objective of fuzzy assignment problem is to find the least assignment fuzzy cost (maximum fuzzy profit) of workers with varying degree of skills to job. To attain the objective in this article, an approach involving modified best candidate method has been used to solve Hexagonal fuzzy assignment problem. To order the hexagonal fuzzy numbers Robust's Ranking technique is applied. We examine a numerical example by using new method and compute by existing two methods. Also we compare the optimal solutions among this new method and two existing method.The proposed method is a systematic procedure, easy to apply for solving fuzzy assignment problem.
\end{abstract}

Keywords - Fuzzy assignment problem, Hexagonal fuzzy Number, Defuzzification technique, Hungarian Method, Best Candidate Method, Modified Best Candidate Method.

\section{Introduction}

The fuzzy set theory was put forward by Zadeh [20] in the year 1965. For the past six decades researchers gave more attention to the set fuzzy theory. It may be applied in the fields like operations research, control theory, neural networks, management science, finance etc. In industry assignment problem (AP) plays a vital role. To deduct the optimal assignment which minimizes the assigning cost is the main goal of AP. The following are assumptions made in AP

$\square$ Each person can be assigned to exactly one job

$\square$ Each person can do at most one job.

As a special case, this article discusses the algorithm to solve using fuzzy parameters with Hexagonal fuzzy costs $C i j H$. Fuzzy Assignment problem have been studied by many researchers. Chen[6] proposed basics theorem and discussed FAP which considers all persons have same skills. Wang[17] proposed an algorithm to solve a FAP where the cost was estimated according to the quality of the job. Mukherjee and Basu [14] transformed the FAP in to crisp AP by applying Yager's ranking technique and solved it. Huang \& Xu [10] developed an algorithm for fuzzy assignment problems with constraints on qualification. Dhanasekar et al.[8] 
applied haar ranking technique in Hungarian algorithm for solving FAP. Khalid et al. [12] used diagonal optimal approach to obtain conventional AP The author used Robust's Ranking technique to defuzzify the FAP and optimal solution is found by Using Ones Assignment problem.[8]In 2012 Hlayel introduced a new method, Best Candidate Method (BCM) of electing the candidates among the other and finding the solution for the optimization problems. [7]Furthermore with slight modifications Hlayel extended the method and proposed Modified Best Candidate method [9] In 2016, BCM was implemented to solve Fuzzy Assignment Problems.

In this paper Hexagonal Fuzzy number has been newly introduced along with the alpha cut operations of arithmetic function principles using addition, subtraction and multiplication has been Ordering the fuzzy numbers plays a vital role in optimization problems. Very few methods are there for Ordering of Hexagonal Numbers. In this article, Robust's ordering technique is used for ordering the Hexagonal fuzzy numbers. Since it depends upon the utmost values of $\alpha$ - cut of hexagonal fuzzy number not on the membership function form. In this article the Hexagonal Fuzzy optimal solution of the HxFAP is obtained by applying Modified Best Candidate method, Best Candidate Method and classical Hungarian Method . A comparative analysis is made by the author among the methods and a conclusion is drawn on which method gives the optimum solution.

\section{PRELIMINARIES}

\section{A.Fuzzy Sets:}

If $\mathrm{X}$ is a collection of objects denoted generically by $\mathrm{x}$, then the fuzzy set $\mathrm{A}$ in $\mathrm{X}$ is a set of ordered pairs $\tilde{A}=\left\{\left(x, \mu_{\tilde{A}}(x)\right), \mid x \in X\right\}$ is called the membership function of $\mathrm{x}$ in A that maps $\mathrm{X}$ to the membership space $\mathrm{M}$ (When $\mathrm{M}$ contains only the two points 0 and $1, \tilde{A}$ is non fuzzy and $\mu_{\tilde{A}}(x)$ is identical to the characteristic function of a nonfuzzy set). The range of the membership function is a subset of the nonnegative real numbers whose supremum is finite.

B.Normal Fuzzy Set:

A fuzzy set $A$ of the universe of discourse $X$ is called a normal fuzzy set implying that there exist at least one $\mathrm{x} \varepsilon \mathrm{X}$ such that $\mu \mathrm{A}(\mathrm{x})=1$.

C. $\alpha-$ cut :

The $\alpha$ Cut of a $\alpha$ level set of a fuzzy set $\tilde{A}$ is a set consisting of those elements of the universe $\mathrm{X}$ whose membership values exceed the threshold level $\alpha$

$$
\tilde{A}_{\alpha}=\left\{x \mid \mu_{\tilde{A}}(x) \geq \alpha\right\}
$$

\section{Triangular Fuzzy Number:}

For a triangular fuzzy number $\mathrm{A}(\mathrm{x})$, it can be represented by $\mathrm{A}(\mathrm{a}, \mathrm{b}, \mathrm{c} ; 1)$ with membership function $\mu(\mathrm{x})$ given by

$$
\mu(x)= \begin{cases}\frac{(x-a)}{(b-a)}, & a \leq x \leq b \\ \frac{(c-x)}{(c-b)}, & b \leq x \leq c \\ 0 & \text { otherwise } \\ \text { where } \mathrm{a} \leq \mathrm{b} \leq \mathrm{c}\end{cases}
$$

\section{E.Trapezoidal Fuzzy Number:}

For a triangular fuzzy number $\mathrm{A}(\mathrm{x})$, it can be represented by $\mathrm{A}(\mathrm{a}, \mathrm{b}, \mathrm{c}, \mathrm{d} ; 1)$ with membership function $\mu(\mathrm{x})$ given by 
where $\mathrm{a} \leq \mathrm{b} \leq \mathrm{c} \leq \mathrm{d}$

$$
\mu(x)=\left\{\begin{array}{cc}
\frac{(x-a)}{(b-a)}, & a \leq x \leq b \\
1 & b \leq x \leq c \\
\frac{(d-x)}{(d-c)}, & c \leq x \leq d \\
0 & \text { otherwise }
\end{array}\right.
$$

F.Hexagonal Fuzzy Number :

A fuzzy number $\tilde{A}_{H}$ is a hexagonal fuzzy number denoted by $\tilde{A}_{H}\left(a_{1}, a_{2}, a_{3}, a_{4}, a_{5}, a_{6}\right)$ where $a_{1}, a_{2}, a_{3}, a_{4}, a_{5}, a_{6}$ are real numbers and its membership function $\mu_{\tilde{A}}(x)$ is given below

$$
\mu(x)=\left\{\begin{array}{rl}
0 & x<a_{1} \\
\frac{1}{2} \frac{\left(x-a_{1}\right)}{\left(a_{2}-a_{1}\right)}, & a_{1} \leq x \leq a_{2} \\
\frac{1}{2}+\frac{1}{2}\left(\frac{x-a_{2}}{a_{3}-a_{2}}\right), & a_{2} \leq x \leq a_{3} \\
1 & a_{3} \leq x \leq a_{4} \\
1-\frac{1}{2}\left(\frac{x-a_{4}}{a_{5}-a_{4}}\right) & , a_{4} \leq x \leq a_{5} \\
\frac{1}{2}\left(\frac{a_{6}-x}{a_{6}-a_{5}}\right) & , a_{5} \leq x \leq a_{6} \\
0 & x>a_{6}
\end{array}\right.
$$

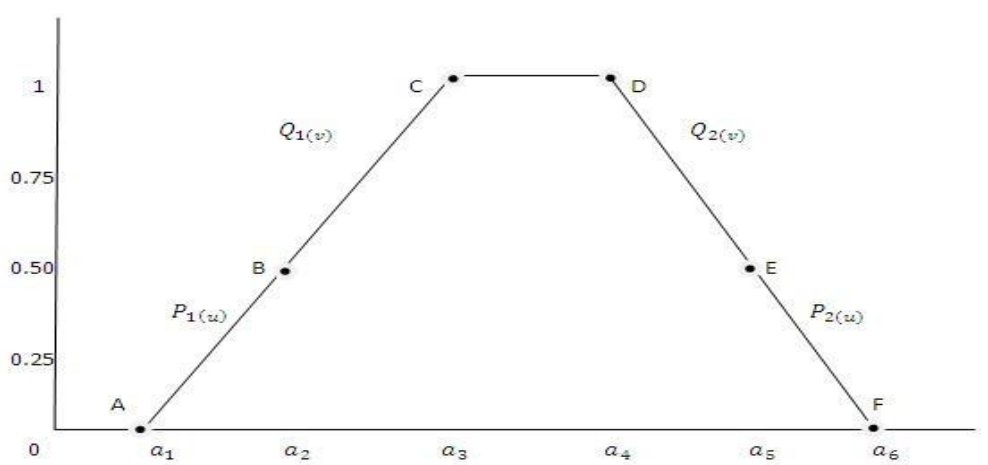

An Hexagonal fuzzy number denoted by $\tilde{A}_{H}$ is defines as $\tilde{A}_{w}\left(P_{1}(u), Q_{1}(v), Q_{2}(v), P_{1}(u)\right)$ for $u \in[0,0.5]$ and $v \in[0.5, w]$ where,

(i) $P_{1}(u)$ is a bounded left continuous non decreasing function over $[0,0.5]$.

(ii) $Q_{1}(v)$ is a bounded left continuous non decreasing function over [0.5,w].

(iii) $Q_{1}(v)$ is a bounded left continuous non decreasing function over [w, 0.5$]$.

(iv) $P_{2}(u)$ is a bounded left continuous non decreasing function over $[0.5,0]$. 


\section{G.Alpha Cut :}

The classical set $\tilde{A}_{\alpha}$ called alpha cut set is the set of elements whose degree of membership is the set of elements whose degree of membership in $\tilde{A}_{H}=\left(a_{1}, a_{2}, a_{3}, a_{4}, a_{5}, a_{6}\right)$ is no less than $\alpha, \mathrm{B}$ it is defined as

$$
\begin{gathered}
A_{\alpha}=\left\{x \in X / \mu_{\tilde{A}_{H}}(x) \geq \alpha\right. \\
=\left\{\begin{array}{lr}
{\left[P_{1}(\alpha), P_{2}(\alpha)\right]} & \text { for } \alpha \in[0,0.5) \\
{\left[Q_{1}(\alpha), Q_{2}(\alpha)\right]} & \text { for } \alpha \in[0.5,1]
\end{array}\right.
\end{gathered}
$$

H.Alpha cut Operations: all $\alpha \in[0,1]$

If we get crisp interval by $\alpha$ cut operations Interval $\mathrm{A}_{\alpha}$ shall be obtained as follows for

Consider $Q_{1}(x)=\alpha$

Similarly consider $Q_{2}(x)=\alpha$

$$
\begin{gathered}
\frac{1}{2}+\frac{1}{2}\left(\frac{x-a_{2}}{a_{3}-a_{2}}\right)=\alpha \\
x=2 \alpha\left(a_{3}-a_{2}\right)-a_{3}+2 a_{2} \\
Q_{1}(\alpha)=2 \alpha\left(a_{3}-a_{2}\right)-a_{3}+2 a_{2}
\end{gathered}
$$

This implies

$$
\begin{gathered}
1-\frac{1}{2}\left(\frac{x-a_{4}}{a_{5}-a_{4}}\right)=\alpha \\
x=-2 \alpha\left(a_{5}-a_{4}\right)+2 a_{5}-2 a_{4}+a_{4} \\
Q_{2}(\alpha)=-2 \alpha\left(a_{5}-a_{4}\right)+2 a_{5}-2 a_{4}+a_{4}
\end{gathered}
$$

$\left[Q_{1}(\alpha), Q_{2}(\alpha)\right]=\left[2 \alpha\left(a_{3}-a_{2}\right)-a_{3}+2 a_{2},-2 \alpha\left(a_{5}-a_{4}\right)+2 a_{5}-2 a_{4}+a_{4}\right]$

Consider $P_{1}(x)=\alpha$

Similarly $P_{2}(x)=\alpha$

$$
x=2 \alpha\left(a_{2}-a_{1}\right)+a_{1}=P_{1}(\alpha)
$$

$$
\begin{gathered}
x=-2 \alpha\left(a_{6}-a_{5}\right)+a_{6}=P_{2}(\alpha) \\
{\left[P_{1}(\alpha), P_{2}(\alpha)\right]=\left[2 \alpha\left(a_{2}-a_{1}\right)+a_{1}=-2 \alpha\left(a_{6}-a_{5}\right)+a_{6}\right]}
\end{gathered}
$$

Therefore

$$
\begin{gathered}
A_{\alpha}=\left\{\begin{array}{cc}
{\left[P_{1}(\alpha), P_{2}(\alpha)\right]} & \text { for } \alpha \in[0,0.5) \\
{\left[Q_{1}(\alpha), Q_{2}(\alpha)\right]} & \text { for } \alpha \in[0.5,1]
\end{array}\right. \\
{\left[\begin{array}{c}
\left.2 \alpha\left(a_{2}-a_{1}\right)+a_{1},=-2 \alpha\left(a_{6}-a_{5}\right)+a_{6}\right]
\end{array}\right.} \\
A_{\alpha}=\left\{\begin{array}{c}
\text { for } \alpha \in[0,0.5) \\
{\left[2 \alpha\left(a_{3}-a_{2}\right)-a_{3}+2 a_{2},-2 \alpha\left(a_{5}-a_{4}\right)+2 a_{5}-2 a_{4}+a_{4}\right]}
\end{array}\right. \\
\text { for } \alpha \in[0.5, w]
\end{gathered}
$$

E.Operations of Hexagonal Fuzzy Number:

Following are the two operations that can be performed on hexagonal fuzzy numbers,

Suppose $\tilde{A}_{H}=(a, b, c, d, e, f)$ and $\tilde{B}_{H}=(p, q, r, s, t, u)$ are two hexagonal fuzzy numbers then Addition and Subtraction of two Hexagonal fuzzy numbers :

$$
\begin{gathered}
\tilde{A}+\tilde{B}=(a+b+c+d+e+f)+(p+q+r+s+t+u) \\
=(a+p, b+q, c+r, d+s, e+t, f+u) \\
\tilde{A}-\tilde{B}=(a-b-c-d-e-f)-(p-q-r-s-t-u) \\
=(a-p, b-q, c-r, d-s, e-t, f-u)
\end{gathered}
$$

F.Defuzzification:

Defuzzification is the process of finding singleton value (crisp value) which represents the average value of the Hexagonal Fuzzy numbers. Here Robust's Ranking technique is used to defuzzify the Hexagonal Fuzzy numbers because of its simplicity and accuracy. 


\section{G.Robust Ranking Technique:}

Robust's ranking technique which satisfy compensation, linearity, and additively properties and provides results which are consist human intuition. If ã is a fuzzy number then the Robust Ranking is defined by

$$
R(\tilde{a})=\int_{0}^{1} 0.5\left(a_{L}^{\alpha}, a_{R}^{\alpha}\right) d \alpha
$$

where $\left(a_{L}^{\alpha}, a_{R}^{\alpha}\right)$ is the $\alpha$-level cut of fuzzy number $\tilde{a}$

The Robust's Ranking technique for hexagonal fuzzy number is :

$$
\begin{gathered}
R(\tilde{a})=\int_{0}^{1} 0.5\left\{\left[P_{1}(\alpha), P_{2}(\alpha)\right],\left[Q_{1}(\alpha), Q_{2}(\alpha)\right]\right\} d \alpha \\
R(\tilde{a})=\int_{0}^{1} 0.5\left\{\left[2 \alpha\left(a_{2}-a_{1}\right)+a_{1},-2 \alpha\left(a_{6}-a_{5}\right)+a_{6}\right],\left[2 \alpha\left(a_{3}-a_{2}\right)-a_{3}\right.\right. \\
\left.\left.+2 a_{2},-2 \alpha\left(a_{5}-a_{4}\right)+2 a_{5}-2 a_{4}+a_{4}\right]\right\} d \alpha
\end{gathered}
$$

This method is for Ranking the objective values. The Robust ranking index $R(\tilde{a})$ gives the representative value of fuzzy number ã.

\section{A.Assignment Problem :}

\section{PROBLEM Formulation}

The Assignment problem (AP) can be stated in the form of $\mathrm{n} \times \mathrm{n}$ cost matrix $\left(C_{i j}\right)_{n x n}$ of real numbers as given in table 1

Table 1. Assignment cost

\begin{tabular}{|l|l|l|l|l|}
\hline $\begin{array}{l}\text { Job } \rightarrow \\
\text { Person } \downarrow\end{array}$ & $\begin{array}{l}\text { Job } \\
1\end{array}$ & $\begin{array}{l}\text { Job } \\
2\end{array}$ & $\begin{array}{l}\text { Job } \\
k\end{array}$ & $\begin{array}{l}\text { Job } \\
n\end{array}$ \\
\hline Person 1 & $\mathrm{C}_{11}$ & $\mathrm{C}_{12}$ & $\mathrm{C}_{1 \mathrm{k}}$ & $\mathrm{C}_{1 \mathrm{n}}$ \\
\hline Person $k$ & $\mathrm{C}_{\mathrm{k} 1}$ & $\mathrm{C}_{\mathrm{k} 2}$ & $\mathrm{C}_{\mathrm{kk}}$ & $\mathrm{c}_{\mathrm{kn}}$ \\
\hline Person $n$ & $\mathrm{C}_{\mathrm{n} 1}$ & $\mathrm{C}_{\mathrm{n} 2}$ & $\mathrm{c}_{\mathrm{nk}}$ & $\mathrm{c}_{\mathrm{nn}}$ \\
\hline
\end{tabular}

Mathematically assignment problem can be stated as:

$$
\operatorname{Min} Z=\sum_{i=1}^{i=n} \sum_{j=1}^{j=n} c_{i j} x_{i j}
$$


Subject to :

$$
(A P)= \begin{cases}\sum_{i=1}^{i=n} x_{i j}=1, & j=1,2, \ldots \ldots \ldots \ldots n \\ j=n & i=1,2, \ldots \ldots \ldots \ldots n\end{cases}
$$

where $x_{i j}=\left\{\begin{array}{c}1, \text { if the } i^{\text {th }} \text { person assign the } j^{\text {th }} \text { job } \\ 0, \text { otherwise }\end{array}\right.$

Suppose there are $n$ jobs to be performed and $n$ persons are available for doing these jobs. Assume that each person can do one job at a time and each job can be assigned to one person only.

\section{B.Fuzzy Assignment Problem:}

Let $\tilde{C} i j$ be the triangular fuzzy numbers cost (payment) if $j t h$ job is assigned to $p t h$ person (see table). The problem is to find an assignment $x i j$ so that the total cost for performing all the jobs is minimum.

Table 1. Fuzzy Assignment cost

\begin{tabular}{|c|c|c|c|c|}
\hline $\begin{array}{l}\text { Job } \rightarrow \\
\text { Person } \downarrow\end{array}$ & $\begin{array}{l}\text { Job } \\
1\end{array}$ & $\begin{array}{l}\text { Job } \\
2\end{array}$ & $\begin{array}{l}J o b \\
k\end{array}$ & $\begin{array}{l}J o b \\
n\end{array}$ \\
\hline Person 1 & $\widetilde{C_{11}}$ & $\widetilde{C_{12}}$ & $\widetilde{C_{1 k}}$ & $\widetilde{C_{1 n}}$ \\
\hline Person $k$ & $\widetilde{C_{k 1}}$ & $\widetilde{C_{k 2}}$ & $\widetilde{C_{k k}}$ & $\widetilde{C_{k n}}$ \\
\hline Person $n$ & $\widetilde{C_{n 1}}$ & $\widetilde{C_{n 2}}$ & $\widetilde{C_{n k}}$ & $\widetilde{C_{n n}}$ \\
\hline
\end{tabular}

The chosen Fuzzy Assignment Problem (FAP) may be formulated into the following fuzzy linear programming problem:

$$
\begin{aligned}
& \operatorname{Min} Z=\sum_{i=1}^{i=n} \sum_{j=1}^{j=n} \widetilde{C_{l j}} x_{i j} \\
& \text { Subject to : } \\
& (A P)= \begin{cases}\sum_{i=1}^{i=n} x_{i j}=1, & j=1,2, \ldots \ldots \ldots \ldots n \\
j=n & i=1,2, \ldots \ldots \ldots \ldots n\end{cases} \\
& \text { where } x_{i j}=\left\{\begin{array}{c}
1, \text { if the } i^{\text {th }} \text { person assign the } j^{\text {th }} \text { job } \\
0, \text { otherwise }
\end{array}\right.
\end{aligned}
$$

III. ALGORITHM

A.Algorithm for Assignment Problem using Modified Best Candidate Method:

Our method is based on determination of the best candidates then elimination the

x. unwanted one in order to minimize the number of solution combinations to decide the optimal 
solution [Hlayel (2012)]. However, we can notice that the solution approach using this method as one of LAP methods is divide into two phases. We will describe each phase and clarify the new modifications. The first Phase, is to elect the best candidates through choosing the prime candidate and its alternative in each row depending on the objective function (maximum or minimum value) then elect one candidate for the columns that have no candidate. There are no new modifications in this phase and the solution findings steps are as follows:

Step1: Prepare the matrix. If the matrix is unbalanced, we balance it and we would not use the added row or column candidates in our solution process.

Step2: Determination of the best candidate, it is used for minimization problems (minimum cost) or maximization problem (maximum profit): Elect the best two candidates in each row, if the candidate repeated more than one times elect it also. Check the columns that not have candidates and elect one candidate from them, if the candidate repeated more than one time elect it also.

The second phase, will introduce the following steps:

a. At the end of phase one an index matrix is produced that shows the position for each candidate.

b. Find the direct combinations and calculating the cost for each.

c. Check the unused candidates, by finding the possible candidates for them then calculate the cost for each.

d. Find the optimal solution according to the objective function.

\section{B.Algorithm to solve Hexagonal fuzzy assignment problem with Modified BCM:}

Step 1: First test whether the given fuzzy cost matrix of an fuzzy assignment problem is a balanced/unbalanced. If not change this unbalanced assignment problem by adding the dummy row (s) / column(s) and the values for the entries are zero. If it is a balanced one

then go to step 2. If it is an unbalanced one then convert it into a balanced one and then go to step2.

Step 2: Replace the cost matrix Hexagonal fuzzy number.

Step 3: Defuzzify the fuzzy cost by using Robust's ranking method

Step 4: Replace Hexagonal numbers by their respective ranking indices.

Step 5: Apply Modified BCM to determine the best combination to produce the lowest total weight of the costs, where elect the best two candidates in each row, if the candidate repeated more than one times elect it also. Check the columns that not have candidates and elect one candidate from them, if repeated more than once elect them.

Step 6: Construct an index matrix and find the direct combination. Calculate cost for each combination. Check for unused candidates, find the possible candidates for them and calculate cost for them also. Now find optimal solution from all the combinations.

\section{C.Algorithm for Best Candidates Method (BCM) has the following Solution Steps}

Step1: From the matrix with the Fuzzy Assignment Costs. Balance the unbalanced matrix and don't use the added row or column candidates in our solution procedure.

Step2: The best candidates are selected by choosing minimum cost for minimization problems and maximum cost for maximization problems. Select the best two candidates in each row, if the candidate is repeated more than two times select it also. Check the columns that not have candidates and select one candidate for them, if the candidate is repeated more than one time select it also.

Step3: The combinations are found by determining only one candidate for each row and column starting from the row that have least candidates and delete that row and column if there is situation that has no candidate for some rows or columns, select directly the best available candidate. Repeat step $3(1,2)$ by determining the next candidate in the row that started from. 
The total sum of candidates for each combination is computed and compared to determine the best combinations that give the optimal solution.

D.Algorithm to solve fuzzy assignment problem with BCM

Step 1: First test whether the given fuzzy cost matrix of an fuzzy assignment problem is a balanced one or not If not change this unbalanced assignment problem by adding the number of dummy row (s) / column(s) and the values for the entries are zero. If it is a balanced one (i.e, number of persons are equal to the number of works) then go to step 2 . If it is an unbalanced one then convert it into a balanced one and then go to step 2.

Step 2: Replace the cost matrix Cij with linguistic variables by triangular or trapezoidal fuzzy numbers.

Step 3: Defuzzify the fuzzy cost by using Robust's ranking method.

Step 4: Replace Hexagonal numbers by their respective ranking indices.

Step 5: Apply BCM to determine the best combination to produce the lowest total weight of the costs, where is one candidate for each row and column.

Step 6: Select the row with the smallest cost candidate from the chosen combination. Then allocate the demand and the supply as much as possible to the variable with the least unit cost in the selected row or column. Also, we should adjust the supply and demand by crossing out the row/column to be then assigned to zero. If the row or column is not assigned to zero, then we check the selected row if it has an element with lowest cost comparing to the determined element in the chosen combination, then we select it.

IV Numerical Example

To illustrate the proposed method a fuzzy assignment problem is solved by using the proposed method. Consider the following Hexagonal Fuzzy Assignment Problem

\begin{tabular}{|l|l|l|l|}
\hline & Machine 1 & $\begin{array}{l}\text { Machine } \\
2\end{array}$ & Machine 3 \\
\hline Jo & $(3,7,11,15$, & $(3,5,7,9$, & $(11,14,17,2$ \\
b 1 & $19,24)$ & $10,12)$ & $1,25,30)$ \\
\hline Jo & $(3,5,7,9,10$ & $(5,7,10,1$ & $(7,9,11,14,1$ \\
b2 & $, 12)$ & $3,17,21)$ & $8,22)$ \\
\hline Jo & $(7,9,11,14$, & $(2,3,4,6$, & $(5,7,8,11,14$ \\
b3 & $18,22)$ & $7,9)$ & $, 17)$ \\
\hline
\end{tabular}

\section{Solution:}

In Conformation to model the fuzzy assignment problem can be formulated as:

$$
\operatorname{Min}\left\{\mathrm{R}(3,7,11,15,19,24) x_{11}+R(3,5,7,9,10,12) x_{12}+R(11,14,17,21,25,30) x_{13}+\right.
$$

$R(3,5,7,9,10,12) x_{21}+R(5,7,10,13,17,21) x_{22}+R(7,9,11,14,18,22) x_{23}+$

$R(7,9,11,14,18,22) x_{31}+R(2,3,4,6,7,9) x_{32}+R(5,7,8,11,14,17) x_{33}$

Subject to

$$
\begin{gathered}
x_{11}+x_{12}+x_{13}=1 \\
x_{21}+x_{22}+x_{23}=1 \\
x_{31}+x_{32}+x_{33}=1
\end{gathered}
$$

and

$$
\begin{gathered}
x_{11}+x_{21}+x_{31}=1 \\
x_{12}+x_{22}+x_{32}=1 \\
x_{13}+x_{23}+x_{33}=1 \\
\text { where } x_{i j} \in[0,1]
\end{gathered}
$$

Now we calculate $R(3,7,11,15,19,24)$ by applying Robust's ranking method. The membership 
function of the hexagonal fuzzy number $(3,7,11,15,19,24)$ is

$$
\mu(x)=\left\{\begin{array}{cc}
\frac{1}{2} \frac{(x-3)}{4}, & 3 \leq x \leq 7 \\
\frac{1}{2}+\frac{1}{2}\left(\frac{x-7}{4}\right), & 7 \leq x \leq 11 \\
1 & , 11 \leq x \leq 15 \\
1-\frac{1}{2}\left(\frac{x-15}{4}\right) & , 15 \leq x \leq 19 \\
\frac{1}{2}\left(\frac{24-x}{5}\right) & , 19 \leq x \leq 24 \\
0 & x>24
\end{array}\right.
$$

\begin{tabular}{|c|c|c|c|}
\hline & M1 & M2 & M3 \\
\hline J1 & 23 & 10 & 39 \\
\hline J2 & 10 & 24 & 27 \\
\hline J3 & 27 & 10.5 & 15 \\
\hline
\end{tabular}

Alpha cut of the fuzzy number $(3,7,11,15,19,24)$ is,

$$
\left[P_{1}(\alpha) P_{2}(\alpha)\right]=[2 \alpha=[8 \alpha+3,-10 \alpha+24]
$$

Alpha cut of the fuzzy number $(3,7,11,15,19,24)$ is,

$$
\begin{gathered}
{\left[Q_{1}(\alpha), Q_{2}(\alpha)\right]=\left[2 \alpha\left(a_{3}-a_{2}\right)-a_{3}+2 a_{2},-2 \alpha\left(a_{5}-a_{4}\right)+2 a_{5}-a_{4}\right.} \\
=[2 \alpha(11-7)-11+14,-2 \alpha(19-15)+19(2)-15] \\
=[8 \alpha-3,-8 \alpha+23] \\
{\left[P_{1}(\alpha) P_{2}(\alpha)\right]=\left[2 \alpha\left(a_{2}-a_{1}\right)+a_{1},-2 \alpha\left(a_{6}-a_{5}\right)+a_{6}\right.} \\
=[2 \alpha(7-3)+3,-2 \alpha(24-19)+24] \\
=[8 \alpha+3,-10 \alpha+24] \\
\left(a_{\alpha}^{L}, a_{\alpha}^{U}\right)=\left[\left[P_{1}(\alpha) P_{2}(\alpha)\right],\left[Q_{1}(\alpha), Q_{2}(\alpha)\right]\right]=([8 \alpha+3,-10 \alpha+24],[8 \alpha-3,-8 \alpha+23]) \\
R(3,7,11,15,19,24)=\int_{0}^{1} 0.5\{[8 \alpha+3,-10 \alpha+24],[8 \alpha-3,-8 \alpha+23]\} d \alpha \\
=\int_{0}^{1} 0.5(-2 \alpha+47) d \alpha=23
\end{gathered}
$$

Similarly the Robust's Ranking indices of the fuzzy cost $\mathrm{a}_{\mathrm{ij}}$ is calculated as follows:

$$
\begin{gathered}
R\left(\tilde{a}_{12}\right)=10 R\left(\tilde{a}_{13}\right)=39 R\left(\tilde{a}_{21}\right)=10 R\left(\tilde{a}_{22}\right)=24 \\
R\left(\tilde{a}_{23}\right)=27 \quad R\left(\tilde{a}_{31}\right)=27 \quad R\left(\tilde{a}_{32}\right)=10.5 \quad R\left(\tilde{a}_{33}\right)=15
\end{gathered}
$$

We replace these values for their corresponding $\mathrm{a}_{\mathrm{ij}}$ in which result in a convenient assignment problem in the linear programming problem.

\begin{tabular}{|c|c|c|c|}
\hline & M1 & M2 & M3 \\
\hline J1 & 23 & 10 & 39 \\
\hline J2 & 10 & 24 & 27 \\
\hline J3 & 27 & 10.5 & 15 \\
\hline
\end{tabular}

\section{Method 1:}

We solve it by modified best candidate method to get the following optimal solution.

Phase 1 :Elect Candidates

Step 1:The matrix is Balanced, where the number of rows is equal to the number of columns as shown in table 1 .

Table 1:Person-Job assignment Profit matrix after balance

Step 2 : Elect the best Candidates as shown in table 
Table 2: Best Candidates Determination Matrix

\begin{tabular}{|c|c|c|c|}
\hline & M1 & M2 & M3 \\
\hline J1 & 23 & 10 & (39) \\
\hline J2 & 10 & 24 & 27 \\
\hline J3 & 27 & 10.5 & 15 \\
\hline
\end{tabular}

Phase 2 : Obtain the BCM Combinations.

a. Draw the following index matrix (Table 3) showing the position of each candidate

Table 3 : Best Candidate Combination Position Matrix.

\begin{tabular}{|c|c|c|c|}
\hline & M1 & M2 & M3 \\
\hline $\mathbf{J 1}$ & A1 & - & A3 \\
\hline $\mathbf{J 2}$ & - & B2 & B3 \\
\hline $\mathbf{J 3}$ & C1 & - & C3 \\
\hline
\end{tabular}

From the above table we obtain the solution set $\{\mathrm{A} 1, \mathrm{~A} 3, \mathrm{~B} 2, \mathrm{~B} 3, \mathrm{C} 1, \mathrm{C} 3\}$.

b. The direct combinations for all the candidates from the solution set and calculate the cost for each:

Combination $1:\{\mathrm{A} 1, \mathrm{~B} 2, \mathrm{C} 3\}=23+24+15=62$

Combination $2:\{\mathrm{C} 1, \mathrm{~B} 2, \mathrm{~A} 3\}=27+24+39=90$

c. Check for unused candidates in the solution set $\{\mathrm{B} 3\}$, then find the possible combinations and calculate cost for each:

Combination $3:\{\mathrm{A} 1, \mathrm{~B} 3\}$ then we add to them $\mathrm{C} 2$ and become $\{\mathrm{A} 1, \mathrm{~B} 3, \mathrm{C} 2\}=23+27+10.5$ $=60.5$

Combination $4:\{\mathrm{C} 1, \mathrm{~B} 3\}$ then we add to them A2 and become $\{\mathrm{A} 2, \mathrm{~B} 3, \mathrm{C} 1\}=10+27+27=64$ $\mathrm{d}$. Find the optimal solution according to the objective function (maximum of minimum cost): In our case it is combination number 2 (modified-BCM solution).

Job J1 $\rightarrow$ Machine M3

Job J2 $\rightarrow$ Machine M2

Job J3 $\rightarrow$ Machine M1 
Method 2 :

We now solve it by using Best Candidate Method

\begin{tabular}{|c|c|c|c|}
\hline & M1 & M2 & M3 \\
\hline J1 & 23 & 10 & 39 \\
\hline J2 & 10 & 24 & 27 \\
\hline J3 & 27 & 10.5 & 15 \\
\hline
\end{tabular}

Solution:

Step 1: Select the best candidates

\begin{tabular}{|l|l|l|l|}
\hline & M1 & M2 & M3 \\
\hline $\mathbf{J 1}$ & 23 & 10 & 39 \\
\hline $\mathbf{J 2}$ & 10 & 24 & 27 \\
\hline $\mathbf{J 3}$ & 27 & 10.5 & 15 \\
\hline
\end{tabular}

Combination 1 is given by

\begin{tabular}{|c|c|c|c|}
\hline & M1 & M2 & M3 \\
\hline $\mathbf{J 1}$ & 23 & 10 & 39 \\
\hline $\mathbf{J 2}$ & 10 & 24 & 27 \\
\hline $\mathbf{J 3}$ & 27 & 10.5 & 15 \\
\hline
\end{tabular}

Combination 2 is given by

\begin{tabular}{|c|c|c|c|}
\hline & M1 & M2 & M3 \\
\hline $\mathbf{J 1}$ & 23 & 10 & 39 \\
\hline $\mathbf{J 2}$ & 10 & 24 & 27 \\
\hline $\mathbf{J 3}$ & 27 & 10.5 & 15 \\
\hline
\end{tabular}

Combination1: $10+10+15=35$

Combination2: $23+24+15=62$ this is the optimal one. 
Job $1 \rightarrow$ Machine 1

Job $2 \rightarrow$ Machine 2

Job $3 \rightarrow$ Machine 3

Method 3 :

Solving it by classical Hungarian method

\begin{tabular}{|c|c|c|c|}
\hline & M1 & M2 & M3 \\
\hline J1 & 23 & 10 & 39 \\
\hline J2 & 10 & 24 & 27 \\
\hline J3 & 27 & 10.5 & 15 \\
\hline
\end{tabular}

Solution:

Step 1: Subtract the smallest entry in each row from all the entries of its row.

\begin{tabular}{|c|c|c|c|}
\hline & M1 & M2 & M3 \\
\hline J1 & 13 & 0 & 29 \\
\hline J2 & 0 & 14 & 17 \\
\hline J3 & 16.5 & 0 & 4.5 \\
\hline
\end{tabular}

Step 2 : Subtract the smallest entry in each row from all the entries of its row.

\begin{tabular}{|c|c|c|c|}
\hline & M1 & M2 & M3 \\
\hline J1 & 13 & 0 & 24.5 \\
\hline J2 & 0 & 14 & 12.5 \\
\hline J3 & 16.5 & 0 & 0 \\
\hline
\end{tabular}

Step 3 : Draw lines through appropriate rows and columns so that all the zero entries of the cost matrix are covered and the minimum number of such lines is used.

\begin{tabular}{|c|c|c|c|}
\hline & M1 & M2 & M3 \\
\hline J1 & 13 & 0 & 24.5 \\
\hline J2 & 0 & 14 & 12.5 \\
\hline J3 & 16.5 & 0 & 0 \\
\hline
\end{tabular}

The optimal Solution is $10+10+15=35$ 


\section{Result and Analysis:}

\begin{tabular}{|c|c|}
\hline Method & Optimal Value \\
\hline Modified BCM & 90 \\
\hline BCM & 62 \\
\hline Hungarian & 35 \\
\hline
\end{tabular}

Therefore the new proposed method gives the maximum profit and its also easy to use.

\section{CONCLUSION}

In this article a new method is introduced called Modified Best candidate method to solve Fuzzy assignment problems. The study gives the reader a clear idea about solvation technique of a Hexagonal fuzzy Assignment problem and also a comparison among the existing methods. This algorithm is productive and easy to comprehensive. As the comparison result, the modified-BCM is the most efficient and accurate comparing to other currently used methods, and it can be easily used in different areas and applications in optimization problems.

\section{[1]L.A.Zadeh, "Fuzzy Sets"}

\section{REFERENCES}

[2] Mohamed Muamer, (2020), Fuzzy Assignment Problems, Journal of Science.

[3] Kadhirvel.K, Balamurugan.K, "Method For Solving Hungarian Assignment Problems Using Triangular And Trapezoidal Fuzzy Number”, International Journal of Engineering Research and Applications (IJERA), ISSN: 2248-9622, Vol. 2, Issue 5, September-October 2012, pp.399-403

[4] Pathinathan. T., Ponnivalavan. K. and Mike Dison. E.," Different Types of Fuzzy Numbers and Certain Properties", Journal of Computer and Mathematical Sciences, Vol.6(11),631-651, November 2015

[5] A.Srinivasan, G.Geetharamani, (2013), Method for solving Fuzzy Assignment Problem using Ones Assignment Method and Robust Ranking Technique, Applied Mathematical Sciences.

[6] P. Rajarajeswari, A.Sahaya Sudha and R.Karthika, "A New Operation On Hexagonal Fuzzy Number”, International Journal of Fuzzy Logic Systems (IJFLS) Vol.3, No3, July 2013, Pg 16 - 22,DOI : 10.5121/ijfls.2013.3302 15

[7] S.Dhanasekar, G.Kanimozhi, A.Manivannan, "Solving Fuzzy Assignment Problems with Hexagonal Fuzzy Numbers by using Diagonal Optimal Algorithm”, International Journal of 
Innovative Technology and Exploring Engineering (IJITEE) ISSN: 2278-3075, Volume-9 Issue-1, November 2019, Pg 55-56, DOI: 10.35940/ijitee.A3912.119119

[8] S.Jayamani, K.Ashwini, N.Srinivasan," Method for Solving Fuzzy Assignment Problem using ones Assignment Method and Robust's Ranking Technique”, Journal of Applied Science and Engineering Methodologies Volume 3, No.2, (2017): Page.488-501

[9] Hlayel Abdallah Ahmad, "The Best Candidates Method for Solving Optimization Problems”, Journal of Computer Science 8 (5): 711-715, 2012, ISSN 1549-3636

[10] Abdallah A. Hlayel, Khulood Abu Maria, "A New Modified Approach Using Best Candidates Method For Solving Linear Assignment Problems", International Journal Of Engineering Science And Technology, ISSN : 0975-5462 Vol. 5 No.05 May 2013, Pg 1137 1142

[11]. S. Krishna Prabha, S. Vimala, (2016), Implementation of BCM for Solving the Fuzzy Assignment Problem with Various Ranking Techniques, Asian Research Journal of Mathematics. 